JOURNAL OF

SYMPLECTIC GEOMETRY

Volume 5, Number 1, 19-41, 2007

\title{
DETERMINING SYMPLECTIC FILLINGS FROM PLANAR OPEN BOOKS
}

\author{
Stephan SchönenBerger
}

We describe a strategy for classifying symplectic fillings of contact structures supported by planar open books. We demonstrate the efficacy of this strategy in certain cases.

Dedicated to Dusa McDuff

\section{Introduction}

The correspondence between contact structures and open books, developed by Giroux in 2000, created a new burst of work in contact topology. Recently, Abbas et al. [1] proved the Weinstein conjecture for contact 3 -manifolds that are supported by planar open books, i.e., open books whose page is a punctured 2-sphere. That this does not solve the Weinstein conjecture in generality was shown by Etnyre [7], who provided the first obstructions for fillable (and hence tight) contact structures to be supported by planar open books. He did so by showing that such fillings can be compactified to a blowup of a ruled symplectic manifold, which proves that, among other things, the intersection form of a filling must have $b_{2}^{+}=0$. Etnyre's proof uses a result by Eliashberg [5], a construction to cap off the boundary of an open book by 2-handles in a symplectic way, thus giving rise to a symplectic cobordism of the 3-manifold to a surface bundle over the circle. Such surface bundles can then be capped off to yield a closed symplectic manifold.

The problem to determine the diffeomorphism types of fillings for contact 3 -manifolds is interesting and intriguing. It was first shown by Eliashberg [4] that any Stein filling of the tight contact 3 -sphere is diffeomorphic to the 4ball. McDuff [18] showed that for contact structures on Lens spaces $L(p, 1)$ that are quotients of a cyclic action on (the unique) tight contact structure on $S^{3}$, there is a unique (up to blowup) diffeomorphism type of fillings 
if $p \neq 4$ and there are two in case $p=4$. Her argument showed that such fillings can be compactified to a ruled symplectic manifold and identified the complement of a filling to be a neighborhood of a symplectic sphere with selfintersection $p>0$. Such configurations of symplectic spheres are unique up to isotopy and this proves her result about the filling itself. Recently, Hind [14] has shown that these fillings are unique up to Stein homotopy. This result was proved for $L(2,1) 3$ years earlier [13] using similar techniques.

Lisca [16] generalized McDuff's result, using a similar line of argument, to contact structures on all Lens spaces that arise as quotients of the tight contact structure on $S^{3}$. To do this, Lisca used a glueing result by McCarthy and Wolfson to construct compactifications of symplectic fillings. Also Ohta and Ono [19] studied diffeomorphism types of fillings for contact structures from Milnor fibers in a similar way. Most of the examples above used a compactification to a ruled surface. Using ad hoc methods to embedd Stein fillings of $T^{3}$ into homotopy $K 3$ surfaces, Stipsicz [22] showed that a Stein filling of the 3 -torus $T^{3}$ with its unique Stein fillable contact structure is homeomorphic to $T^{2} \times D^{2}$.

We follow McDuff's strategy for solving this problem, but use Etnyre's construction to provide compactifications to a ruled surface. Doing this carefully allows to determine the complement of a filling and classify fillings up to diffeomorphism. Determining the complement of the filling is a difficult task. We arrange to do so in certain cases when the contact structure is supported by a planar open book with monodromy consisting of Dehn twists about nonintersecting curves.

Each of the small Seifert fibered spaces

$$
Y_{n}=M\left(-3 ;-\frac{2 n}{2 n-1},-\frac{2 n}{2 n-1},-\frac{2 n}{2 n-1}\right), n \geq 1
$$

admits two nonisotopic, but contactomorphic contact structures. The strategy above is explained for the case $n=1$ by proving the following theorem; see Theorem 4.3 .

Theorem 1.1. Each of the tight contact structures on the Seifert fiber space $M(-3 ;-2,-2,-2)$ admits a unique symplectic filling, up to blowup and diffeomorphism. In particular, there is a unique Stein filling, up to diffeomorphism.

The proof of this theorem can be extended to a proof of the general case $n \geq 1$; see Theorem 4.4.

Theorem 1.2. Each of the tight contact structures on the Seifert fiber space $Y_{n}$ admits a unique symplectic filling, up to blowup and diffeomorphism. In particular, there is a unique Stein filling, up to diffeomorphism. 


\section{Contact structures and open books}

For an introduction to open books and contact structures, the reader is referred to $[\mathbf{6}, \mathbf{2 0}]$. We assume familiarity with Kirby Calculus, see $[\mathbf{1 2}, \mathbf{2 0}]$.

All 3-manifolds compact, oriented, and all contact structures are positive and cooriented. An open book $(B, \pi)$ for a 3 -manifold $Y$ is given by an oriented link $B \subset Y$ together with a fibration $\pi: Y \backslash B \rightarrow S^{1}$ of its complement with fiber $\pi^{-1}(\theta)=\operatorname{int}\left(F_{\theta}\right)$, the interior of a compact surface $F_{\theta} \subset M$ with oriented boundary $\partial F_{\theta}=B$. We call $F_{\theta}$ a page of the open book and $B$ the binding. Any vector field $v$ transverse to the pages and meridional in a neighborhood of $B$ gives rise to a return map $\phi_{v}: F_{0} \rightarrow F_{0}$, where $F_{0}$ is a page of the open book and $\phi_{v}$ is called the monodromy of the open book.

A contact structure $\xi$ on $Y$ is called compatible with an open book $(F, \phi)$ if $d \alpha$ gives a volume form on the pages $F$ and $\left.\alpha\right|_{\partial F}>0$. We also say such an open book decomposition of $Y$ supports $\xi$. Already Thurston and Winkelnkemper [23] showed that an open book decomposition of an orientable 3-manifold gives rise to a compatible contact structure. Giroux [10] and Torisu [24] observed that this contact structure is unique up to isotopy. Giroux also proved the converse, which makes this relationship most useful.

Theorem 2.1. Every open book decomposition of a 3-manifold supports a contact structure, unique up to isotopy. Any contact structure is supported by an open book.

Two open books $(F, \pi)$ and $\left(F^{\prime}, \pi^{\prime}\right)$ of $Y$ are called isomorphic if there exists a diffeomorphism $\phi: Y \rightarrow Y$ such that $\pi=\pi^{\prime} \circ f$, i.e., $\phi$ takes pages to pages and binding to binding. Notice that contact structures supported by isomorphic open books are contactomorphic.

\section{Constructing planar open books}

A plumbing tree $P$ is a tree where each vertex is endowed with an integer. Replacing each vertex by an unknot such that two are linked exactly once if and only if there is an edge between the corresponding vertices gives a link $L \subset S^{3}$. A 3-manifold $Y$ is obtained from a plumbing tree by doing surgery along $L$ where the surgery coefficient on each component is the integer on the corresponding vertex. A contact manifold is obtained by Legendrian realizing the link and performing contact $(-1)$-surgery along this Legendrian link such that, topologically, the surgery is as given by the coefficients. We aim to prove the following.

Theorem 3.1. Suppose $P$ is a plumbing tree such that each vertex is labeled with a framing coefficient $r$ satisfying $r \leq-\min \{-d,-2\}$, where $d$ is the valence of that vertex. Any Legendrian realization of the surgery diagram 
according to $P$ gives rise to a Stein fillable contact structure that is supported by a planar open book.

Remark 3.2. If there is a bad vertex, i.e., one not satisfying the inequality $r \leq-d$, then there might be no planar open book for a Legendrian realization of the plumbing. For example, the unique Stein fillable contact structure of the Poincaré homology sphere is not supported by a planar open book [7]. Etnyre and Ozbagci $[\mathbf{8}]$ extend the construction below to the case where bad vertices are present and exhibit many minimal genus open books for manifolds that arise from plumbings.

We will gradually build up the proof that constructs these open books explicitly. First, the case of linear trees gives rise to Lens spaces. There, the concept of rolling up is explained. Second, if there is one vertex with valence three, a small Seifert fibered space is obtained and the idea of hooking in a surgery diagram is most easily seen. Third, the two strategies "rolling up" and "hooking in" are used to construct open books for plumbings as in Theorem 3.1. Because these open books will have positive monodromy, they support Stein fillable contact structures. Although not used explicitly, the construction of such open books is inspired by the algorithm presented in [3] that describes how to turn rational contact surgery into a sequence of $( \pm 1)$-contact surgeries.

3.1. Lens spaces. For coprime integers $p>q \geq 1$, consider the continued fraction expansion

$-\frac{p}{q}=a_{1}-\frac{1}{a_{2}-1 /\left(a_{3}-\cdots-1 / a_{k}\right)}=\left[a_{1}, \ldots, a_{k}\right], \quad a_{i} \leq-2, \quad i=1, \ldots, k$.

The Lens space $L(p, q)$ is obtained from a linear plumbing tree with $k$ vertices and labels $a_{1}, \ldots, a_{k}$. By performing a sequence of slam-dunks starting with the rightmost component, one obtains the usual picture of $L(p, q)$ as $(-p / q)$ surgery along an unknot. Instead, we will perform handle slides. Starting with the leftmost component $K_{1}$, slide the right neighbor $K_{2}$ over $K_{1}$. Thus $K_{2}$ links $K_{1}$ exactly $t_{1}=\left(a_{1}+1\right)$ times and has as surgery coefficient $b_{2}=a_{1}+a_{2}+2$. The string $K_{3}, \ldots, K_{k}$ remains unchanged. Now we slide $K_{3}$ over $K_{2}$. Afterwards, $K_{3}$ links $K_{1}$ exactly $\left(a_{1}+1\right)$ times, $K_{2}$ is linked an additional $\left(a_{2}+2\right)$ times and the surgery coefficient is $b_{3}=a_{1}+a_{2}+a_{3}+4$. Continuing similarly, we obtain a new surgery diagram as shown in Figure 1. A sequence of handle slides as just performed is called rolling up a linear tree. For an explicit example, see Figure 2 below.

Theorem 3.3. Any tight contact structure on a Lens space $L(p, q)$ is supported by a planar open book.

Proof. Suppose

$$
-\frac{p}{q}=\left[a_{1}, \ldots, a_{k}\right], \quad a_{i} \leq-2, \quad i=1, \ldots, k
$$




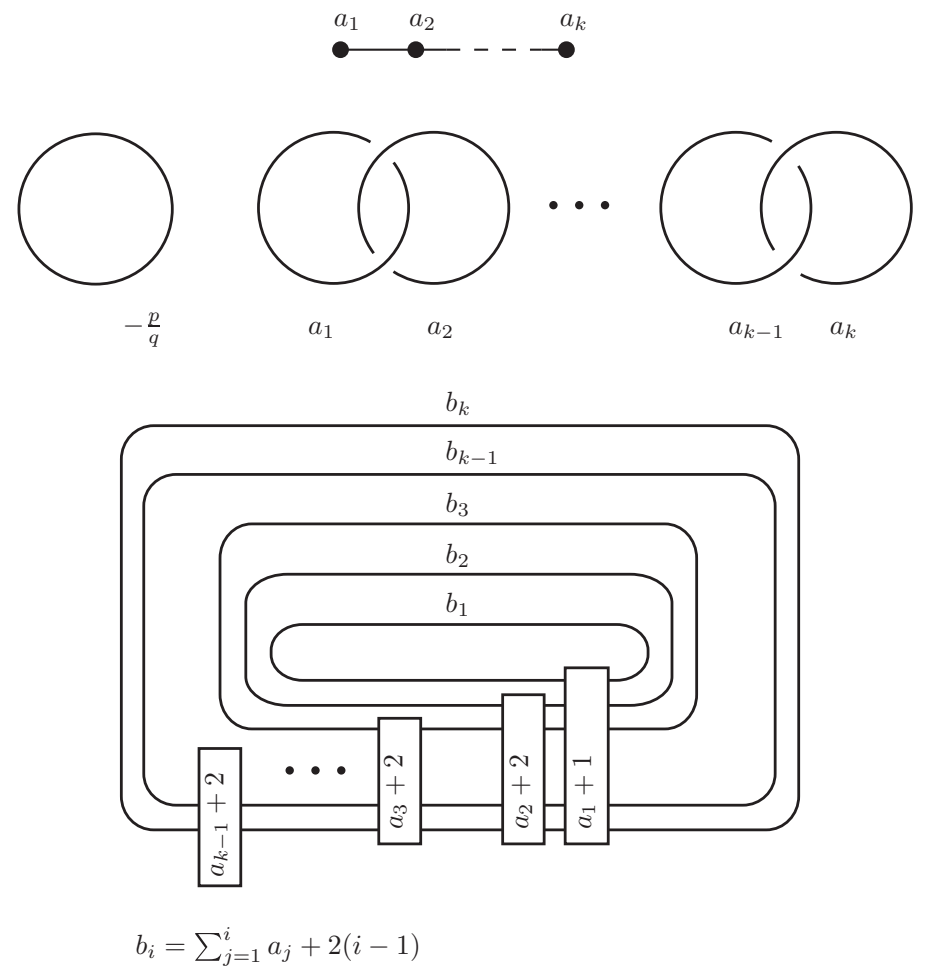

Figure 1. Surgery diagrams for $L(p, q)$ : plumbing along a linear tree, rational and integer surgery, and its rolled-up version.
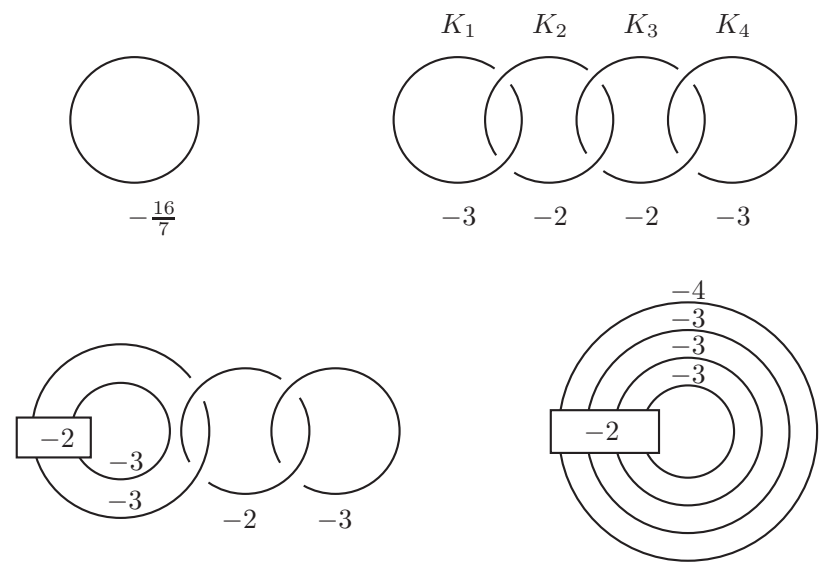

Figure 2. Rolling up the surgery for $L(16,7)$. 
and $K$ is the link in $S^{3}$ obtained from the linear plumbing by rolling up as above, see Figure 1.

There exists an open book of $S^{3}$ such that each component $K_{i}$ of $K$ is homological nontrivially contained in a page and so that the page framing is $r_{i}+1$. Start with the open book given by the positive Hopf fibration $\pi_{+}$of $S^{3}$, which has an annulus as page and the monodromy consists of a positive Dehn twist about its core. Next, we need to arrange for each $K_{i}$ to be contained in a page and that the page framing differs from the framing at hand by 1 . We can realize $K_{1}$ by stabilizing (see Figure 12 in [6]) a parallel copy of the core of a page of $\pi_{+}\left|a_{1}+2\right|$ times. There is not a unique way to do so. We choose one. Proceeding by induction, suppose we have realized $K_{1}, \ldots, K_{i-1}$. To get $K_{i}$, pick a parallel copy of $K_{i-1}$ and stabilize it $\left|a_{i}+2\right|$ times. Eventually, we obtain an open book for a contact structure on $L(p, q)$ by adding a positive Dehn twist along each $K_{i}$ to the monodromy of the open book of $S^{3}$. Since every stabilization is done on a connected component of the boundary of a page, starting with an annulus, the page of the resulting open book is planar. By construction, the monodromy consists solely of positive Dehn twists and thus the resulting open book supports a Stein fillable contact structure on $L(p, q)$.

The number of choices during this construction is exactly the number of tight contact structures on $L(p, q)$, provided through the classification of such by Giroux [9] and Honda [15]. To show that different choices yield different contact structures, notice that Legendrian realizing $K$ gives in particular a Legendrian link in $S^{3}$ such that contact $(-1)$-surgery yields the contact structure supported by the open book constructed above. The contact structures can then be distinguished by using a result of Lisca and Matić [17].

Example 3.4. A rational surgery diagram for the Lens space $L(16,7)$ is shown in Figure 2. Notice that

$$
-\frac{16}{7}=[-3,-2,-2,-3]
$$

This gives the linear plumbing tree which we roll up to obtain the last picture in Figure 2. We exhibit the open book for a contact structure on the left in Figure 3. A Legendrian realization of the corresponding link $K$ is given on the right in Figure 3. Notice that this link is also obtained from contact $\left(-\frac{16}{7}+1\right)$-surgery along a Legendrian unknot with $t b=-1$ by the algorithm described in [3].

3.2. Small Seifert fibered spaces. A 3-manifold obtained by plumbing along a tree as in Figure 4 is called a small Seifert fibered space and is denoted by $M\left(e_{0} ; r_{1}, r_{2}, r_{3}\right)$, where $r_{1}=\left[a_{1}, \ldots, a_{k_{1}}\right], r_{2}=\left[b_{1}, \ldots, b_{k_{2}}\right], r_{3}=$ $\left[c_{1}, \ldots, c_{k_{3}}\right]$. The coefficient $e_{0}=\left\lfloor r_{1}\right\rfloor+\left\lfloor r_{2}\right\rfloor+\left\lfloor r_{3}\right\rfloor$ is called the integral Euler number; see $[\mathbf{1 1}]$ for facts on Seifert fibered spaces. 

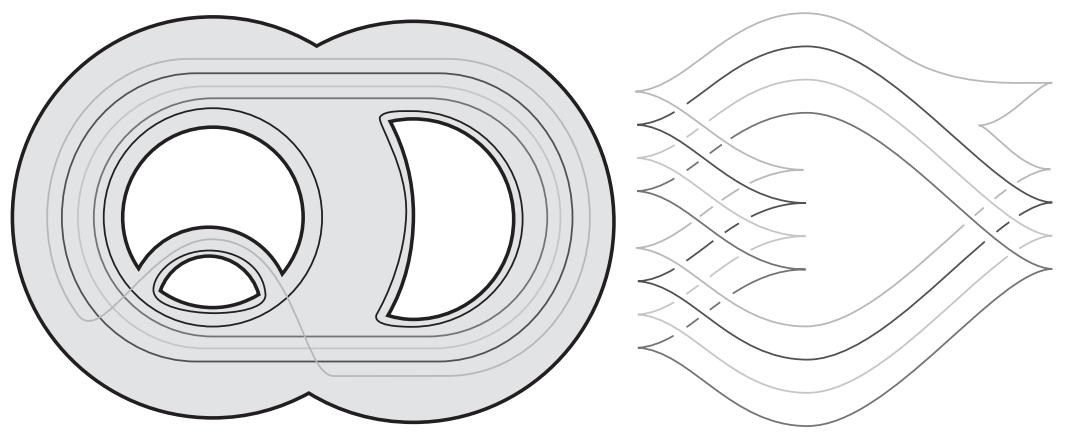

Figure 3. Legendrian realization of the "rolled-up" diagram in Figure 2 (right); a corresponding (abstract) planar open book (left). The monodromy consists of one positive Dehn twist along each curve.

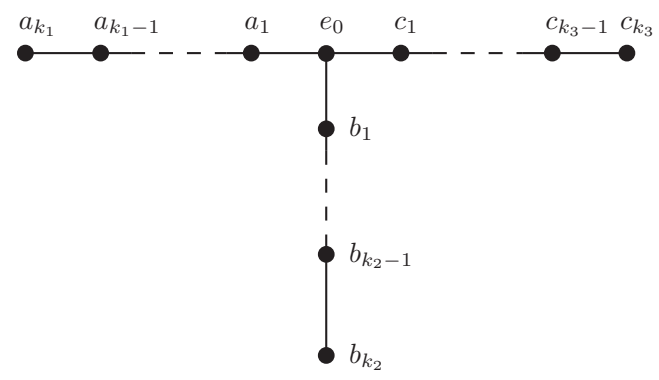

Figure 4. Plumbing diagram for $M\left(e_{0} ; r_{1}, r_{2}, r_{3}\right)$.

Theorem 3.5. Any tight contact structure on $M\left(e_{0} ; r_{1}, r_{2}, r_{3}\right)$ with $e_{0} \leq-3$ is supported by a planar open book.

Proof. Consider a small Seifert fibered space $M\left(e_{0} ; r_{1}, r_{2}, r_{3}\right)$ with $e_{0} \leq-3$ and $r_{1}, r_{2}, r_{3}<-1$. Denote the continued fraction expansions of $r_{i}$ by $r_{1}=\left[a_{1}, \ldots, a_{k_{1}}\right], r_{2}=\left[b_{1}, \ldots, b_{k_{2}}\right]$ and $r_{3}=\left[c_{1}, \ldots, c_{k_{3}}\right]$. A plumbing diagram for $M\left(e_{0} ; r_{1}, r_{2}, r_{3}\right)$ is shown in Figure 4.

As in the case of Lens spaces, first put the link corresponding to the plumbing into a special position. Roll up the linear plumbing tree corresponding to the Lens space $L(p, q)$ with $p, q$ such that

$$
-\frac{p}{q}=\left[a_{k_{1}}, \ldots, a_{1}, e_{0}, c_{1}, \ldots, c_{k_{3}}\right]
$$

to obtain a sequence of knots as pushoffs of each other. Denote by $A_{i}, B_{i}$, $C_{i}$ and $E$ the components corresponding to $a_{i}, b_{i}, c_{i}$ and $e_{0}$, respectively. The sequence of pushoffs starts with, say, $A_{k_{1}}$. In the process of rolling up, observe that a meridian of $E$ will become an unknot that links $E$ and its neighbors $C_{i}$ to the right exactly once. By replacing a neighborhood 
of this unknot with the rolled-up surgery diagram for $L\left(p^{\prime}, q^{\prime}\right)$ with $-\frac{p^{\prime}}{q^{\prime}}=$ $\left[b_{1}, \ldots, b_{k_{2}}\right]$, which is the Lens space obtained from the remaining vertical chain, we hook in an additional branch of the tree. Using Kirby Calculus, one can prove that the surgery thus obtained indeed gives $M\left(e_{0} ; r_{1}, r_{2}, r_{3}\right)$.

Realize this link on an open book of $S^{3}$ as above: Starting with the positive Hopf fibration for $S^{3}$, construct an open book for $L(p, q)$ via stabilizations, as in the proof of Theorem 3.3. When arranging the component $E$ on a page of an open book, one has to stabilize at least once, due to the condition $r \leq-d$. The core curve of this stabilization is taken as a starting point to construct an open book of $L\left(p^{\prime}, q^{\prime}\right)$, so that also components $C_{i}$ are contained on pages of an open book. This is achieved as in Theorem 3.3. Eventually, we obtain a planar open book for a contact structure on $M\left(e_{0} ; r_{1}, r_{2}, r_{3}\right)$, by adding a positive Dehn twist along each of the $A_{i}, B_{i}$, $C_{i}$ and $E$.

To prove that different choices during this construction give rise to different contact structures and the number of such matches the number given by the classification of tight contact structures provided by $\mathrm{Wu}$ [25], is proved as in the proof of Theorem 3.3.

Example 3.6. Take $Y=M(-3 ;-3 / 2,-5 / 3,-5 / 3)$. This manifold has a plumbing diagram as in Figure 5.

First consider the surgery diagram for the Lens space given by the horizontal chain, which is $L(45,19)$; note $-\frac{45}{19}=[-3,-2,-3,-2,-3]$. Pick an unknot $U$ linking the third component corresponding to the central -3 and all the following ones once, and hook in a rolled-up surgery diagram of the Lens space $L(3,2)$, corresponding to the remaining vertical chain. Figure 6 displays a Legendrian realization of this link.

Similar to the previous case, we can realize this link on pages of an open book $S^{3}$ with planar pages by starting with the Hopf fibration of $S^{3}$ and using stabilizations. The hooked-in link requires an additional stabilization for the central component, which can be used as starting point to realize it via stabilizations. We obtain an open book for $Y$ with planar pages, supporting a Stein fillable contact structure. Such an open book is displayed in Figure 7.

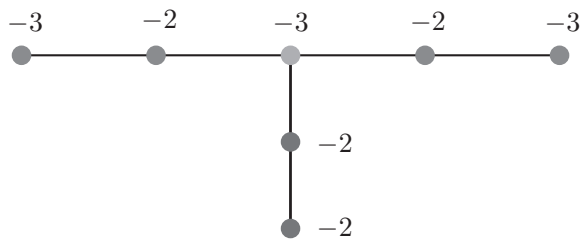

Figure 5. Plumbing diagram for $M(-3 ;-3 / 2,-5 / 3,-5 / 3)$. 


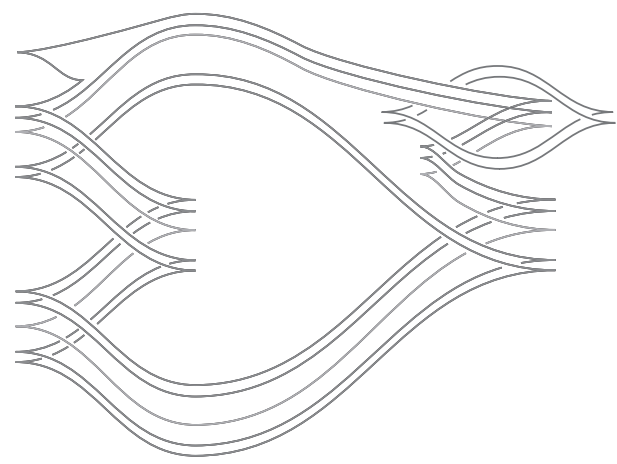

Figure 6. Legendrian realization of the surgery for $M$.

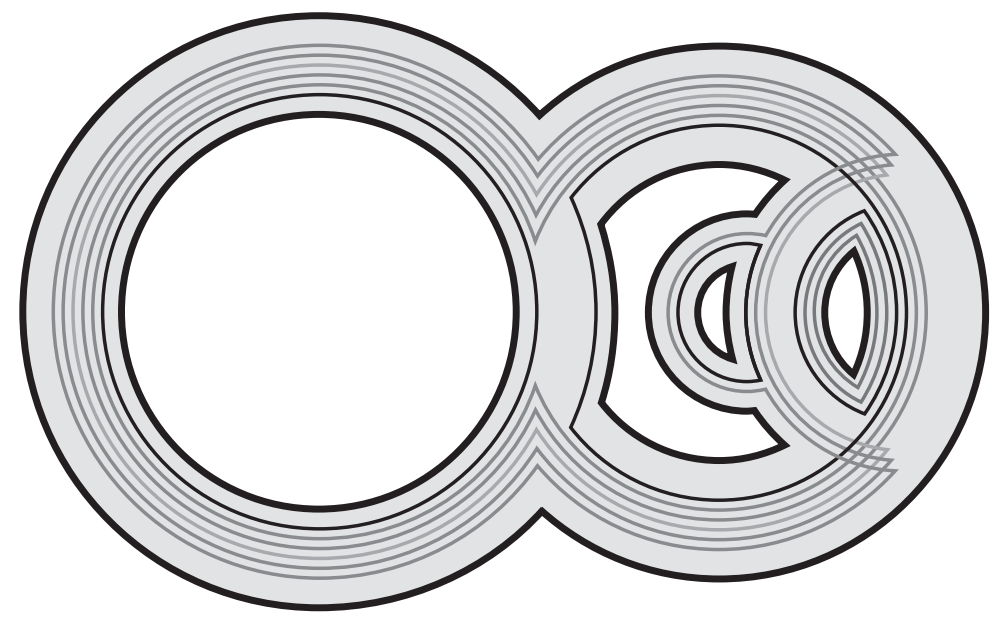

Figure 7. Open book for supporting the contact structure given in Figure 6. The monodromy consists of a positive Dehn twist along each curve.

Proof of Theorem 3.1. Given a 3-manifold $Y$ from a general plumbing tree $P$, pick out a linear tree $P_{1}$. If a branch is separating from a vertex $v$ contained in $P_{1}$, choose another linear tree $P_{2}$ starting at a vertex adjacent to $v$. Inductively, $P$ is split up into a sequence of linear trees $P_{i}$. Construct a special surgery diagram as above. Roll up the diagram for $P_{1}$ and inductively hook in rolled-up diagrams of the remaining ones. Then construct an open book of $S^{3}$ such that each component is contained on a page as above. Starting with the positive Hopf fibration of $S^{3}$, arrange $P_{1}$ to be contained on pages of an open book of $S^{3}$. Suppose a component $V$ corresponding to the vertex $v$ of $P_{i}$, where a new branch $P_{i+1}$ is separating off, is to be arranged. Use the additional stabilization available because of $r \leq-d$ as a starting point to fit the new branch $P_{i+1}$ onto pages of an open book via 
stabilizations. Eventually, get to an open book for a Stein fillable contact structure of $Y$ by adding a positive Dehn twist to the monodromy along each component of the link.

Remark 3.7. Notice that, in general, one cannot prove that all tight contact structures on a plumbing are supported by planar open books. For example, Seifert fibered spaces containing an incompressible torus have infinitely many contactomorphism classes of tight contact structures, but the construction above only gives finitely many. Also, in general, there is no classification of tight contact structures for these spaces available yet.

Still it is possible to prove that different choices in the construction above yield different contact structures, up to isotopy, as before.

\section{Diffeomorphism types of fillings}

This section shows how to use planarity of open books compatible with symplectic fillable contact structures to collect information about the diffeomorphism types of fillings.

We follow Lisca [16], but use planarity of open books to produce embeddings into closed symplectic manifolds, as explained in [7]. The general strategy is made explicit by means of a concrete example.

Consider the small Seifert fibered spaces

$$
Y_{n}=M\left(-3 ;-\frac{2 n}{2 n-1},-\frac{2 n}{2 n-1},-\frac{2 n}{2 n-1}\right), \quad n \geq 1 .
$$

In particular, $Y_{1}=M(-3 ;-2,-2,-2)$ is given by the plumbing diagram in Figure 8.

From the classification of $\mathrm{Wu}[\mathbf{2 5}]$, one concludes the following.

Proposition 4.1. There exist exactly two Stein fillable and hence tight contact structures on $Y_{n}, n \geq 1$, up to isotopy. The two contact structures are contactomorphic.

By Theorem 3.1 and its proof, one obtains immediately the following.
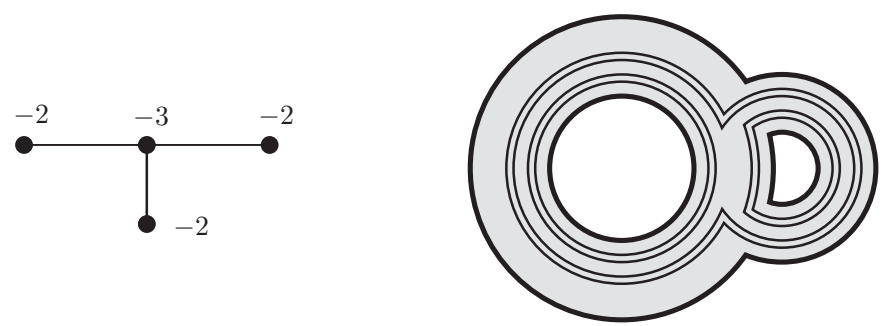

Figure 8. Plumbing picture and a corresponding planar open book for the manifold $Y_{1}$. 
Lemma 4.2. $\left(Y_{n}, \xi\right)$ is supported by the planar open book consisting of a pair of pants with $2 n$ positive Dehn twists about each boundary component, where $\xi$ is any of the two contact structures on $Y_{n}$.

Figure 9(a) gives a picture of the 4-manifold $W$ bounding $Y=Y_{1}$, obtained from the open book in Figure 8, using dotted circles for the 1-handles. By adding handle slides and canceling the 1-handles, one obtains a manifold as shown in Figure 9(b). This verifies directly that $Y=\partial W$ is given by the plumbing in Figure 8. Also, Figure 9(c) gives a Legendrian surgery for $W$ inducing one of the contact structures on $Y$. To see the other, simply rotate this picture $180^{\circ}$, which also proves that these two contact structures are contactomorphic. For the remainder, let $\xi$ denote one of the two contact structures on $Y$.

The aim of this section is to prove the following result.

Theorem 4.3. Any symplectic filling $W^{\prime}$ for $(Y, \xi)$ is diffeomorphic to a smooth blowup of $W$, obtained from the plumbing; see Figure $9(c)$. In particular, there is a unique Stein filling of $(Y, \xi)$ up to diffeomorphism.

The proof takes two steps. In the first step, we compactify $W^{\prime}$ to a closed symplectic manifold $X$ and study its complement in $X$. In the second step we show that the diffeomorphism type of the complement of such a configuration in $X$ is unique.

More generally, the following theorem is proved similarly with more notational effort.

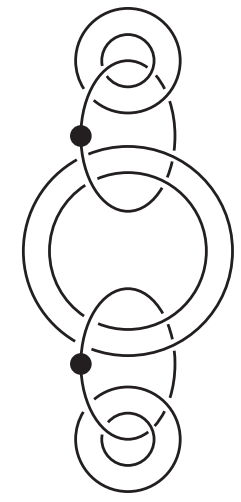

all 2-handles have framing -1

(a)
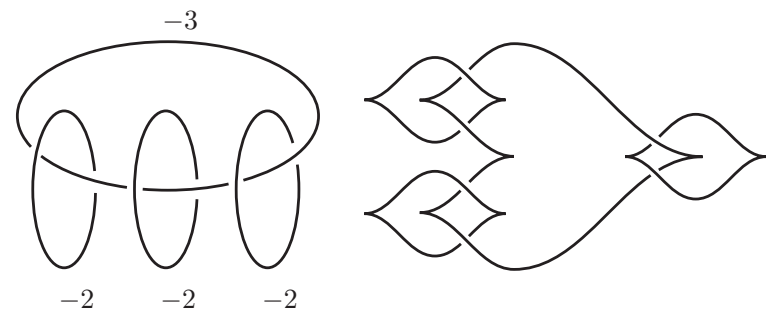

(b) (c)

Figure 9. Kirby pictures for $W$. 
Theorem 4.4. Each of the tight contact structures on the Seifert fiber space $Y_{n}, n \geq 1$, admits a unique symplectic filling, up to blowup and diffeomorphism. In particular, there is a unique Stein filling, up to diffeomorphism.

\section{Compactification of fillings}

First, toward a proof of Theorem 4.3, we study the compactification of a filling to a closed symplectic manifold.

Theorem 5.1. Suppose $W^{\prime}$ is a symplectic filling for $(Y, \xi)$. Then, for some integer $N \geq 1, W^{\prime}$ is diffeomorphic to the complement of a symplectic configuration $\Gamma=C_{0} \cup C_{1} \cup C_{2} \cup C_{3}$ in $X_{N}=\left(S^{2} \times S^{2} \# N \overline{\mathbb{C P}}^{2}\right.$, $\left.\omega\right)$, with $\omega$ a blowup of a symplectic structure on $S^{2} \times S^{2}$.

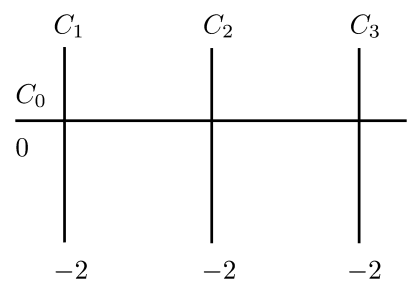

Proof. From Lemma 4.2, the contact structure $\xi$ on $Y$ induced by $W^{\prime}$ is supported by an open book with page $F$, a pair of pants, and monodromy $\phi$ consisting of two positive Dehn twists about three curves, each parallel to one of the boundary components, as shown in Figure 8. We attach Eliashberg handles $H_{i}, i=1,2,3$, one to each of the boundary components and extend $\phi$ by the identity over the resulting 2 -sphere, still calling it $\phi$.

Further notice that $\phi$ is isotopic to the identity. Thus, by adding these handles, we obtain $W^{\prime} \subset W^{\prime \prime}$ with $\partial W^{\prime \prime}=S^{2} \times S^{1}$.

We can symplectically cap off $W^{\prime \prime}$ with an $S^{2} \times D^{2}$. Notice that the resulting closed symplectic manifold contains an embedded symplectic sphere $S_{0}=S^{2} \times\{p\} \subset S^{2} \times D^{2}$ with self-intersection 0 . Thus, from McDuff's theorem, we conclude that $(X, \omega)$ is a blowup of a ruled surface.

Furthermore, the cocore of an $E$-handle $H_{i}$ is a symplectic disk with a neighborhood symplectomorphic to $D^{2} \times D^{2} \subset \mathbb{R}^{4}$ with its standard symplectic structure. Thus, these cocores can be glued to $\{p t\} \times D^{2}$ in the final cap to form a symplectic sphere $S_{i}$. Each $S_{i}$ is disjoint from $S_{j}$ for $i \neq j$ and intersects $S_{0}$ geometrically once. Thus, we obtain a symplectic configuration as in $\Gamma$ and we are left to verify the self-intersection of $S_{i}, i=1,2,3$.

To find these, we only need to find the self-intersections topologically, which can be done via Kirby calculus: Start with the planar open book for $(Y, \xi)$, shown in Figure 8. Adding the $E$-handles amounts to attaching 2 -handles with framing 0 with respect to a page of the open book. Then switching to dotted circle notation gives Figure 10(a). Specify the boundary curves of the cocores, which are denoted by dashed circles in Figure 10, and 


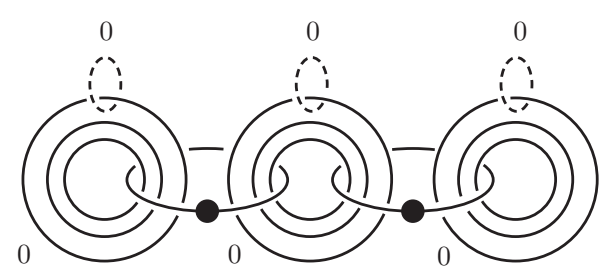

(a) all unlabeled 2-handles have framing -1

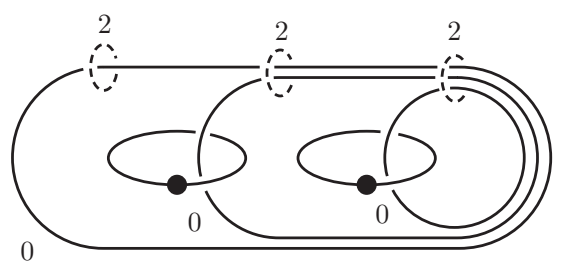

(c)

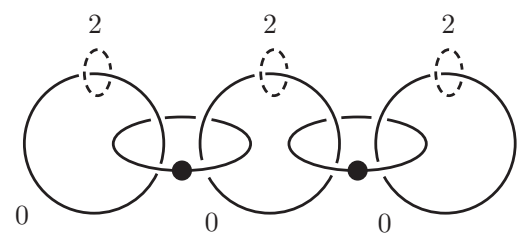

(b)

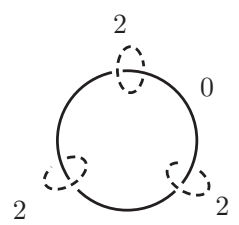

(d)

Figure 10. Calculating the self-intersections of $S_{i}$.

endow them with labels 0 . Now following through the Kirby moves to see the $S^{2} \times S^{1}$ at hand, observe what the labels for the cocores become and these correspond to the self-intersections of the $S_{i}$. To do this, first slide each pair of $(-1)$-framed 2-handles over the 0 -framed 2 -handles to which they are parallel. Thus, these only link the dashed circle specified on that 0 -framed 2-handle once and blowing them down rises label of each dashed circle to 2, as in Figure 10(b). Now slide each 0-framed 2-handle over its neighbors to the right, which gives Figure 10(c). Now we can cancel the 1-handles, obtaining Figure 10(d). From the labels of the dashed circles, we read off the self-intersection number of each $S_{i}$, which is -2 . Notice the change in sign that comes from the fact that we need to turn the handlebody upside down to see the configuration for what it is.

Because the sphere $\tilde{S}$ in the homology class $S_{1}+S_{0}$ has self-intersection 0 and intersects $S_{0}$ exactly one time, the neighborhood of $S_{0} \cup \tilde{S}$ is a punctured $S^{2} \times S^{2}$ and McDuff's result implies that $(X, \omega)$ is symplectomorphic to $\left(S^{2} \times S^{2} \# N \overline{\mathbb{C} P}^{2}, \omega\right)$, with $\omega$ a symplectic structure on $S^{2} \times S^{2}$ blown up. Furthermore, McDuff tells us that we can choose this symplectomorphism to map the sphere $S_{0}$ to $S^{2} \times\{p t\}$.

\section{Complements of the symplectic configuration}

In this section, we study the complement of the symplectic configuration $\Gamma$ obtained in Theorem 5.1. We do this first on the homology level. 
6.1. Homological properties of the configuration $\Gamma$. Recall that the intersection form on $H_{2}\left(S^{2} \times S^{2} \# N \overline{\mathbb{C P}}^{2} ; \mathbb{Z}\right)=\mathbb{Z} \oplus \mathbb{Z} \oplus \oplus_{i=1}^{N} \mathbb{Z}$ is

$$
\left[\begin{array}{ll}
0 & 1 \\
1 & 0
\end{array}\right] \oplus\left(-\mathbb{I}_{N}\right)
$$

where $\left(-\mathbb{I}_{N}\right)$ denotes the negative identity $N \times N$ matrix. We fix a basis $s_{1}=\left[S^{2} \times\{p t\}\right], s_{2}=\left[\{p t\} \times S^{2}\right]$ and $f_{j}, j=1, \ldots, N$, for the homology classes generated by the $\overline{\mathbb{C P}}^{2}$. Pick an almost complex structure $J$ on $X_{N}$ such that $\Gamma$ consists of holomorphic spheres. Expressing the $\left[C_{i}\right]$ in terms of this basis

$$
\begin{aligned}
& {\left[C_{0}\right]=s_{1},} \\
& {\left[C_{i}\right]=\sigma_{i}^{1} s_{1}+\sigma_{i}^{2} s_{2}+\sum_{j=1}^{N} \phi_{i}^{j} f_{j}, \quad i=1, \ldots, 3}
\end{aligned}
$$

we conclude first from $\left[C_{0}\right] \cdot\left[C_{i}\right]=1$ that $\sigma_{i}^{2}=1$ for all $i$. Thus, for the following, we write $\sigma_{i}$ for $\sigma_{i}^{1}$.

We find that, since $\left[C_{i}\right]^{2}=-2$,

$$
2 \sigma_{i}+2-\sum_{j}\left(\phi_{i}^{j}\right)^{2}=0 .
$$

From the adjunction formula $\left\langle c_{1}\left(X_{N}\right),\left[C_{i}\right]\right\rangle=2+\left[C_{i}\right]^{2}$, we learn that

$$
2 \sigma_{i}+2+\sum_{j} \phi_{i}^{j}=0 .
$$

Subtracting equation (6.3) from equation (6.4) gives

$$
\sum_{j} \phi_{i}^{j}+\left(\phi_{i}^{j}\right)^{2}=0 .
$$

Furthermore, since for $i \neq k$ we have $\left[C_{i}\right] \cdot\left[C_{k}\right]=0$,

$$
\sigma_{i}+\sigma_{k}-\sum_{j} \phi_{i}^{j} \phi_{k}^{j}=0 .
$$

Now either equation (6.3) or (6.4) implies

$$
\sigma_{i} \geq-1, \quad i=1, \ldots, 3 .
$$

From equation (6.5), we conclude that

$$
\phi_{i}^{j} \in\{-1,0\}, \quad i=1, \ldots, 3 ; j=1, \ldots, N .
$$

Then, either equations (6.3) or (6.4) implies that for each $i$ there exist

$$
j_{1}^{i}, \ldots, j_{2\left(\sigma_{i}+1\right)}^{i} \in\{1, \ldots, N\}
$$

such that $\phi_{i}^{j}=-1$ if and only if $j=j_{l}^{i}$ for some $l=1, \ldots, 2\left(\sigma_{i}+1\right)$. 
To meet equation (6.6), one first notices that $\sigma_{i}+\sigma_{k} \geq 0$. Furthermore, among the $\left\{j_{l}^{i}\right\}_{l=1}^{2\left(\sigma_{i}+1\right)}$ and $\left\{j_{l}^{k}\right\}_{l=1}^{2\left(\sigma_{k}+1\right)}$ exactly $\sigma_{i}+\sigma_{k}$ are equal. But for this to be possible, one needs that $\sigma_{i}+\sigma_{k} \leq \min \left\{2\left(\sigma_{i}+1\right), 2\left(\sigma_{k}+1\right)\right\}$.

Without loss of generality, we can order the $\left[C_{i}\right]$ such that $\sigma_{1} \leq \sigma_{2} \leq \sigma_{3}$. Then we summarize the calculations above in the following lemma.

Lemma 6.1. In the situation above, there are the following possibilities for the homology classes of $\Gamma=C_{0} \cup C_{1} \cup C_{2} \cup C_{3} \subset X_{N}$.

Let $\sigma_{1}=-1$.

$$
\begin{aligned}
& {\left[C_{0}\right]=s_{1},} \\
& {\left[C_{1}\right]=-s_{1}+s_{2},} \\
& {\left[C_{2}\right]=s_{1}+s_{2}-\sum_{l=1}^{4} f_{j_{l}^{2}},} \\
& {\left[C_{3}\right]=s_{1}+s_{2}-\sum_{l=1}^{4} f_{j_{l}^{3}} .}
\end{aligned}
$$

Without loss of generality, we can assume that $f_{j_{l}^{2}}=f_{j_{l}^{3}}$ if and only if $l=1,2$. Notice that $N \geq 6$.

Let $\sigma_{1}=n$ with $n \geq 0$. Then $\sigma_{2}=n+s$ and $\sigma_{3}=n+t$, where we can assume that $s \leq t$ with $s, t \in\{0,1,2\}$.

$$
\begin{aligned}
& {\left[C_{0}\right]=s_{1},} \\
& {\left[C_{1}\right]=n s_{1}+s_{2}-\sum_{l=1}^{2(n+1)} f_{j_{l}^{1}},} \\
& {\left[C_{2}\right]=(n+s) s_{1}+s_{2}-\sum_{l=1}^{2(n+s+1)} f_{j_{l}^{2}},} \\
& {\left[C_{3}\right]=(n+t) s_{1}+s_{2}-\sum_{l=1}^{2(n+t+1)} f_{j_{l}^{3}} .}
\end{aligned}
$$

Furthermore, note that

$$
\left|\left\{j_{l}^{i}\right\}_{l=1}^{2\left(\sigma_{i}+1\right)} \cap\left\{j_{l}^{k}\right\}_{l=1}^{2\left(\sigma_{k}+1\right)}\right|=\sigma_{i}+\sigma_{k}, \quad i \neq k
$$

and

$$
\max \{0,2 n+s+t-2\} \leq\left|\bigcap_{i=1}^{3}\left\{j_{l}^{i}\right\}_{l=1}^{2\left(\sigma_{i}+1\right)}\right| \leq 2 n+s .
$$

Thus, there are 10 subcases for $\sigma_{1}=n \geq 1$ and 7 in case $\sigma_{1}=0$. 
6.2. Blowing down to a minimal model. We know from McDuff [18] that one can always blow down symplectic spheres with square -1 and hence obtain a minimal symplectic manifold. This is also possible relative to a symplectic configuration. The following lemma generalizes verbatim from [16, Lemma 4.5]. We provide the argument here for completeness.

Lemma 6.2. In the situation of Theorem 5.1, let $J$ be an almost complex structure tamed by $\omega$ such that $\Gamma$ is holomorphic. Then, there exists a holomorphic sphere $S \subset X_{N}$ with square -1 and $[S] \cdot\left[C_{0}\right]=0$. Furthermore, there exists such an $S$ disjoint from $\Gamma$ if and only if there exists a symplectic sphere $S$ of square -1 such that $[S] \cdot\left[C_{i}\right]=0$ for $i=1,2,3$.

Proof. Because $X_{N}$ is obtained from $S^{2} \times S^{2}$ by blowing up, there exists a symplectic sphere $S \subset X_{N}$ of square -1 such that $[S] \cdot\left[C_{0}\right]=0$. By $[\mathbf{1 8}$, Lemma 2.1], the homology class $[S]$ is either represented by an embedded sphere or a cusp-curve $S_{1} \cup \cdots \cup S_{l}$, i.e., a union of (not necessarily embedded) holomorphic spheres. In the first case, the first part of the lemma is proved. In the second case, notice that

$$
[S] \cdot\left[C_{0}\right]=\left(\left[S_{1}\right]+\cdots+\left[S_{l}\right]\right) \cdot\left[C_{0}\right]=0
$$

which, by positivity of intersection, implies that

$$
\left[S_{i}\right] \cdot\left[C_{0}\right]=0 \text { for } i=1, \ldots, l .
$$

Therefore,

$$
\left[S_{i}\right]^{2} \leq-1 \quad \text { for } \quad i=1, \ldots, l
$$

But

$$
\begin{aligned}
1 & =\chi(S)+[S] \cdot[S] \\
& =\left\langle c_{1}\left(X_{N}\right),[S]\right\rangle \\
& =\sum_{i=1}^{l}\left\langle c_{1}\left(X_{N}\right),\left[S_{i}\right]\right\rangle \\
& =\sum_{i=1}^{l} \chi\left(S_{i}\right)+\left[S_{i}\right] \cdot\left[S_{i}\right]
\end{aligned}
$$

implies that $\left[S_{i}\right] \cdot\left[S_{i}\right]=-1$ for at least one index $i \in\{1, \ldots, l\}$. By the adjunction formula [18], choosing $S=S_{i}$ is an embedded sphere.

If $[S]$ is orthogonal to all the classes $\left[C_{i}\right]$, then, by positivity of intersections, $S$ must be disjoint from $\Gamma$, which proves the second part of the lemma.

Thus, blowing down all the existing -1 spheres yields the following. 
Proposition 6.3. There exists a sequence of blowdowns $\left(X_{N}, \Gamma\right)$ to $\left(S^{2} \times\right.$ $\left.S^{2}, \Gamma^{\prime}\right)$, where $\Gamma^{\prime}$ is given as follows. (The boxes in the right-hand side of the figure denote the appropriate number of intersections, as in Lemma 6.1.)
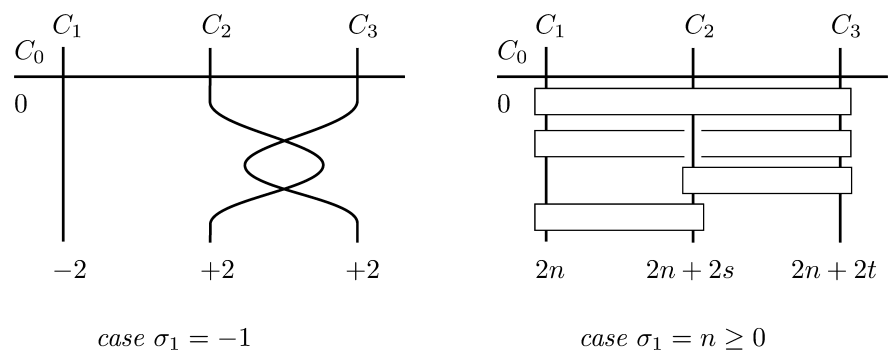

We like to argue that the symplectic configuration thus obtained is unique. For the case where $\sigma_{1} \geq 0$, this follows immediately from the following

Proposition 6.4. Suppose $S_{i}, i=1,2$, are embedded symplectic 2 -spheres in $\left(S^{2} \times S^{2}, \omega\right)$ for some symplectic structure $\omega$, representing the same homology class $\left[S_{i}\right]=n s_{1}+s_{2}$, such that $\left[S_{1}\right] \cdot\left[S_{2}\right]=2 n$. Here denote by $s_{1}$ and $s_{2}$ the homology classes $\left[S^{2} \times\{p t\}\right]$ and $\left[\{p t\} \times S^{2}\right]$ in $H_{2}\left(S^{2} \times S^{2} ; \mathbb{Z}\right)$. Then the two spheres are isotopic.

Proof. Choose an almost complex structure that makes the spheres holomorphic. If $n<0$, then the two spheres coincide by positivity of intersections. If $n=0$, then again by positivity of intersections, the two spheres either coincide or are disjoint. In the latter case, $S_{i}=\left\{p_{i}\right\} \times S^{2}$ for two distinct points in the first factor. Any path on that sphere joining $p_{1}$ and $p_{2}$ provides an isotopy. In the case $n>0$, notice that $S_{1}$ and $S_{2}$ intersect in $2 n$ points (counting multiplicity). The moduli space of spheres in this class is a manifold of real dimension $2\left(c_{1}\left(\left[n s_{1}+s_{2}\right]\right)-1\right)=2(2 n+1)$. Keeping $S_{1} \cap S_{2}$ fixed, there exists a path $\gamma:[0,1] \rightarrow S^{2} \times S^{2}$ with $\gamma(t) \cap S_{1}=\gamma(0)$, $\gamma(t) \cap S_{2}=\gamma(1)$ and such that for each $t$ there is a holomorphic sphere $S_{t}$ through the $2 n+1$ points $S_{1} \cap S_{2} \cup \gamma(t)$. This provides an isotopy from $S_{1}$ to $S_{2}$.

The case where $\sigma_{1}=-1$ is more difficult due to the presence of a symplectic sphere of square -2 . In this case, we can use a construction by Abreu [2] who shows that this case is a symplectomorphic to a standard Hirzebruch surface.

Proof of Theorem 4.3. Starting with one of these unique configurations in $S^{2} \times S^{2}$, we can blow up back to the situation in Theorem 5.1. Doing this in all possible ways and proving, the complements of the configuration $\Gamma$ thus obtained are diffeomorphic is now possible by using Kirby Calculus. 

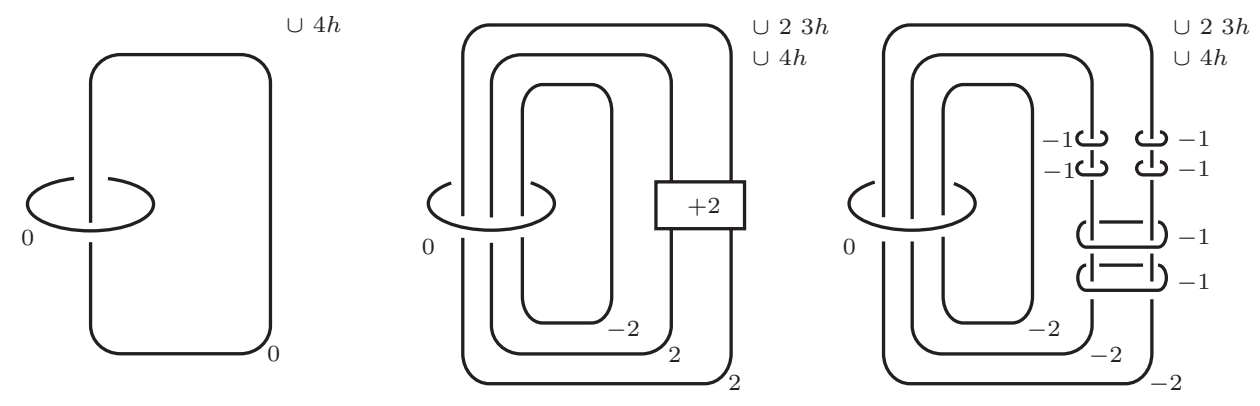

Figure 11. Blowing back up to $\Gamma$ from the minimal model in case $\sigma_{1}=-1$.

We show this process of blowing up in one situation; see Figure 11. All other cases are obtained similarly; see Figures 12 and 13. Start with the usual handle decompostion for $S^{2} \times S^{2}$, shown in the leftmost part of Figure 11 . Add two canceling 2/3-handlepairs and slide the 2 -handles over the 0 -framed 2-handle. Thus, we find three unlinked 2-handles linking once an unknot and all components have framing 0 . Now pick one of these three handles and subtract it from the unknot. When adding the other two 2handles to the unknot, we find the middle part of Figure 11. We can blow up the crossings and then each component individually until each of the three components has framing -2 . This is shown in the rightmost part of Figure 11. Theorem 4.3 follows from the following theorem.

Theorem 6.5. Suppose $W^{\prime}$ is the complement of $\Gamma \subset X_{N}$ obtained by blowing up a minimal model from Proposition 6.3. Then $W^{\prime}$ is diffeomorphic to a smooth blowup of $W$ as in Figure $9(a)$.

Proof. We begin by examining all possible ways to blow up a minimal model as described by Proposition 6.3 to get back to the original configuration $\Gamma \subset X_{N}$. For the case $\sigma_{1}=-1$, there is only one way to do this, shown in the rightmost part of Figure 11. The cases where $\sigma_{1}=n \geq 0$ are shown in Figures 12 and 13.

Immediately from Figures 11-13, one realizes that all the complements are diffeomorphic up to blowup. Suppose there is a component, coming from the blowup procedure, that links all three (-2)-framed 2-handles once. We can slide such a component over the 0 -framed 2-handle and free it from the picture, without changing its framing from -1 . Thus, such components can be blown down. Then, by again sliding the components coming from the blowup procedure about the 0 -framed 2 -handle, one can get one picture from the other. Such handle slides do not change the diffeomorphism type of the complement. 
$(0,0,0)$

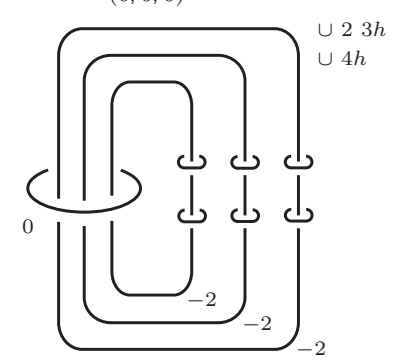

$(0,1,1)(a)$

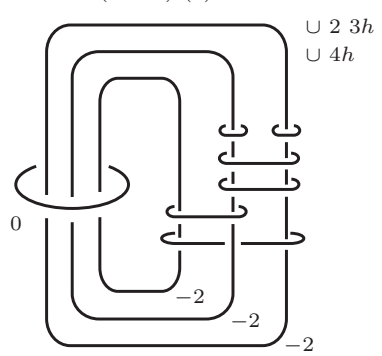

$(0,0,1)$

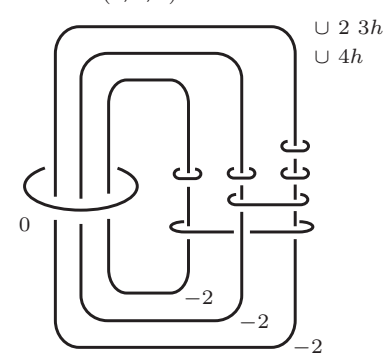

$(0,1,1)(b)$

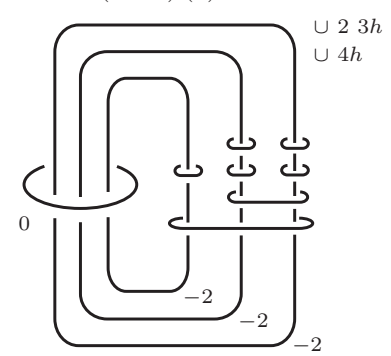

$(0,2,2)$

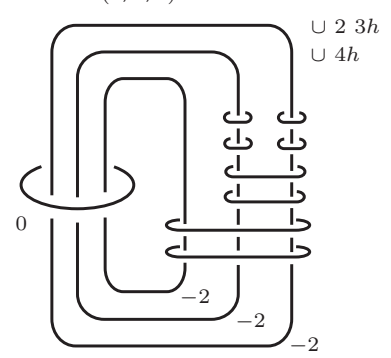

$(0,0,2)$

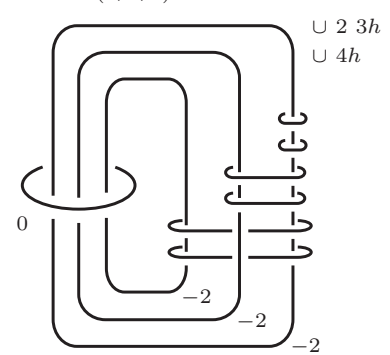

$(0,1,2)$

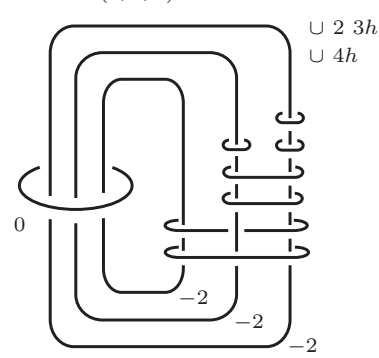

Figure 12. Blowing back up to $\Gamma$ from the minimal model in case $\sigma_{1}=0$. (All unlabeled 2-handles have framing -1 . The triple of numbers on top denotes $\left(\sigma_{1}, \sigma_{2}, \sigma_{3}\right)$.)

This shows that there is at most one filling up to diffeomorphism and blowup. Since we already provided one, the theorem is proved.

We finish by explicitly showing, for one case, how to find the filling that was described in Figure 9. To get a handle on $W^{\prime}=X_{N} \backslash \operatorname{nbhd}(\Gamma)$, we do the following. Put all the framings of 2-handles coming from $\Gamma$ and the blowup circles in brackets $\langle\cdot\rangle$. Then specify cocores of the blowup circles and label them 0 . Now we can use Kirby calculus on the $\langle\cdot\rangle$-framed handles to simplify the picture. Eventually turning the handlebody upside down gives 

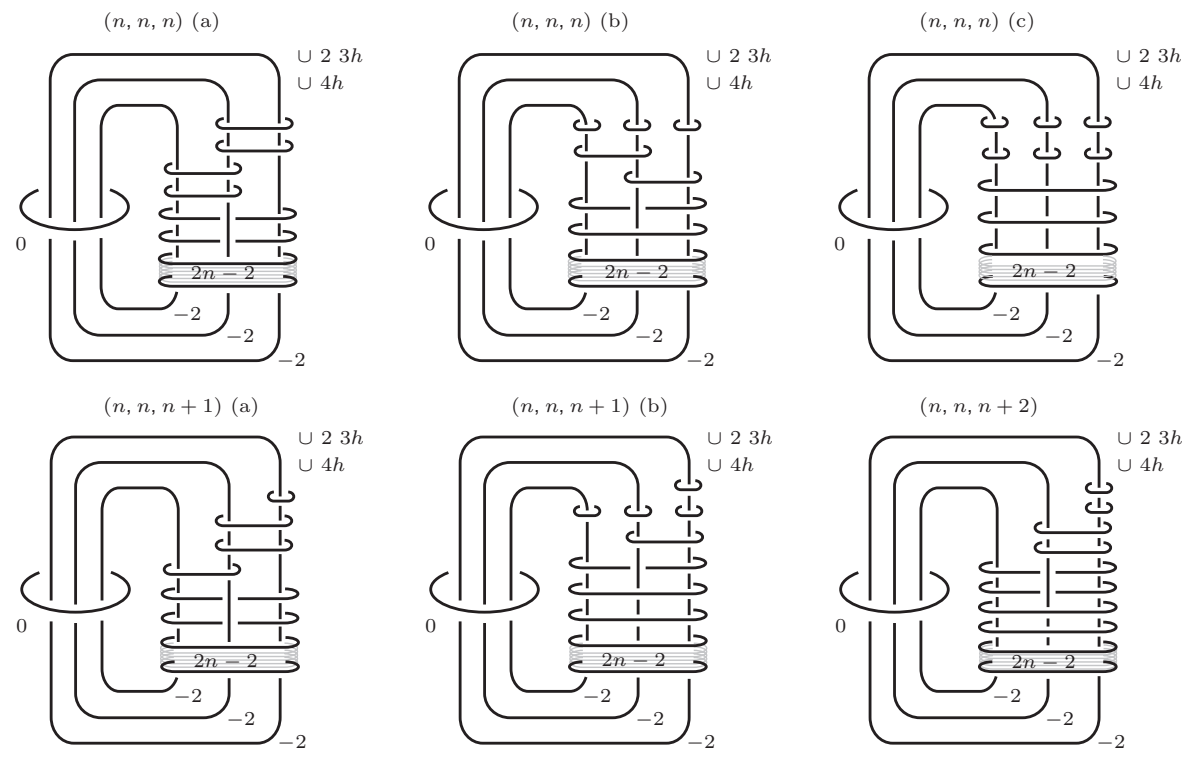

$(n, n+1, n+1)($ a)
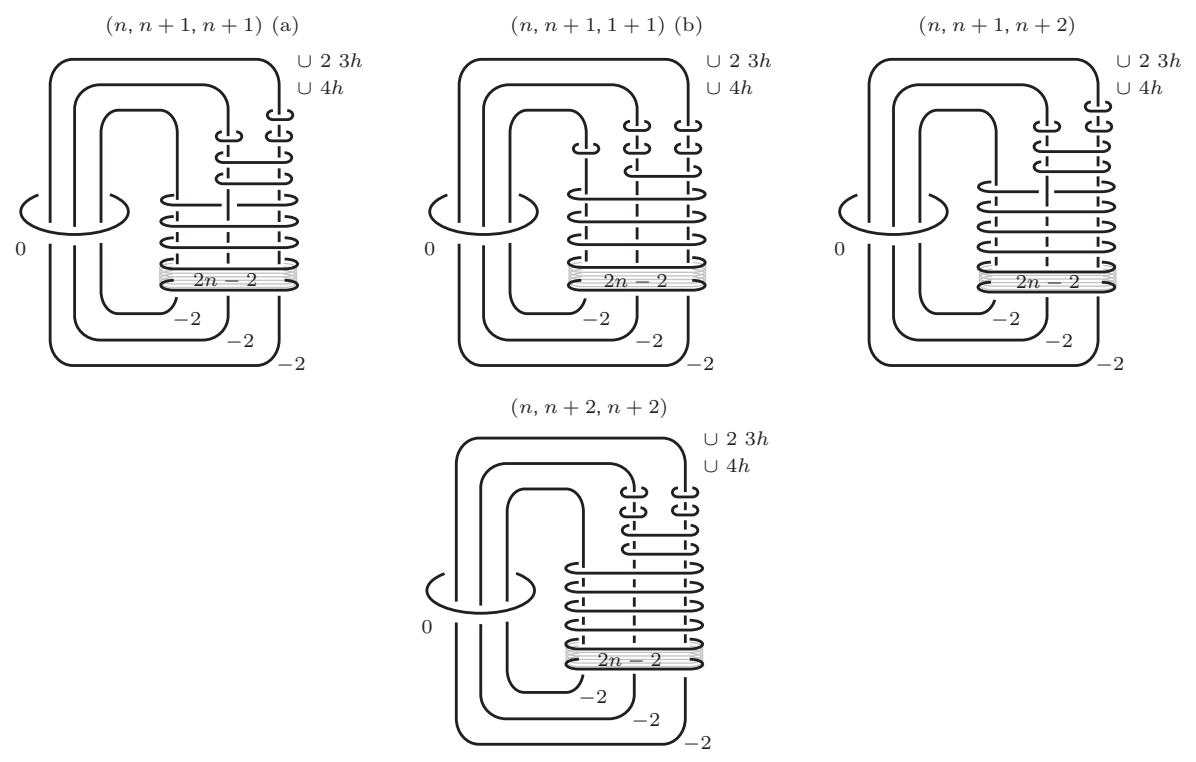

Figure 13. Blowing back up to $\Gamma$ from the minimal model in case $\sigma_{1} \geq 1$. (All unlabeled 2-handles have framing -1.)

a picture of $W^{\prime}$; see [12]. This is explained in Figure 14. Starting with the diagram on top, first blow down all the $\langle-1\rangle$-framed 2-handles. This gives the second diagram. Then, sliding the $\langle 0\rangle$-framed handles over its neighbors to the right yields the third diagram. In there, the two rightmost $\langle 0\rangle$-framed 

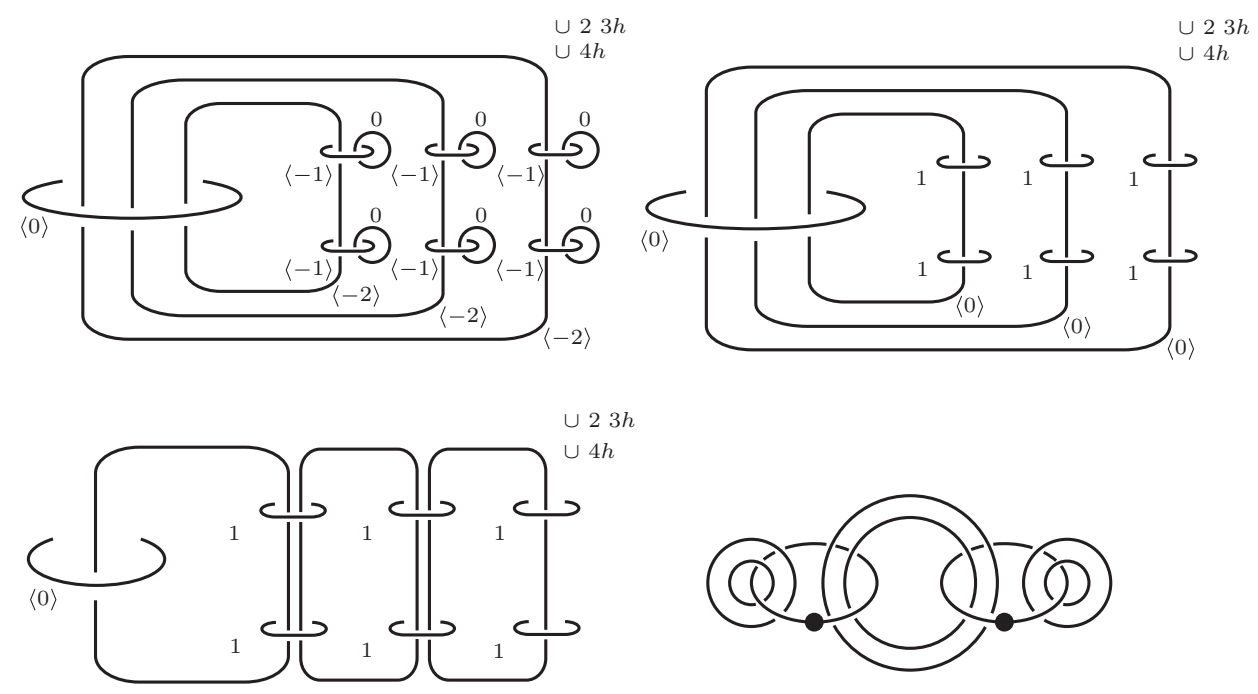

$\langle 0\rangle$

$\langle 0\rangle$

$\langle 0\rangle$

Figure 14. Recreating $W$.

handles bound canceling 3-handles. When turning this handlebody upside down, those 3 -handles become 1 -handles and then erasing all the $\langle\cdot\rangle$-framed 2-handles gives a diagram for $W$, see the last picture. This is exactly what is shown already in Figure 9.

\section{References}

[1] C. Abbas, K. Cieliebak, and H. Hofer. The Weinstein conjecture for planar contact structures in dimension three. Comment. Math. Helv., 80(4) (2005), 771-793.

[2] M. Abreu, Topology of symplectomorphism groups of $S^{2} \times S^{2}$, Invent. Math. 131(1) (1998), 1-23.

[3] F. Ding, H. Geiges and A. Stipsicz, Surgery diagrams for contact 3manifolds, Turkish J. Math. 28(1) (2004), 41-74.

[4] Y. Eliashberg, Filling by holomorphic discs and its applications, in Geometry of low-dimensional manifolds, 2 (Durham, 1989), 45-67, London Math. Soc. Lecture Note Ser., 151, Cambridge University Press, Cambridge, 1990.

[5] Y. Eliashberg, A few remarks about symplectic filling, Geom. Topol. 8 (2004), 277-293 (electronic).

[6] John B. Etnyre. Lectures on open book decompositions and contact structures, in 'Floer homology, Gauge theory, and low-dimensional topology' (Ellwood, David A. (ed.) et al.), Proceedings of the Clay 
Mathematics Institute 2004 summer school, Budapest, Hungary, June 5-26, 2004. Providence, RI: American Mathematical Society (AMS). Cambridge, MA: Clay Mathematics Institute. Clay Mathematics Proceedings 5, 2006, 103-141.

[7] J. Etnyre, Planar open book decompositions and contact structures. Int. Math. Res. Not. 79 (2004), 4255-4267.

[8] J. B. Etnyre and B. Ozbagci. Open books and plumbings, 2006.

[9] E. Giroux, Structures de contact en dimension trois et bifurcations des feuilletages de surfaces, Invent. Math. 141(3) (2000), 615-689.

[10] E. Giroux, 'Géométrie de contact: de la dimension trois vers les dimensions supérieures', in Proceedings of the International Congress of Mathematicians, Vol. II (Beijing, 2002), pp. 405-414, Higher Ed. Press, Beijing, 2002.

[11] R. Gompf, Handlebody construction of Stein surfaces, Ann. of Math. (2) 148(2) (1998), 619-693.

[12] R. Gompf and A. Stipsicz, '4-manifolds and Kirby calculus', Graduate Studies in Mathematics, 20, American Mathematical Society, Providence, RI, 1999.

[13] R. Hind, Holomorphic filling of $\mathbf{R P}^{3}$, Commun. Contemp. Math. 2(3) (2000), 349-363.

[14] R. Hind, Stein fillings of lens spaces, Commun. Contemp. Math. 5(6) (2003), 967-982.

[15] K. Honda, On the classification of tight contact structures. I, Geom. Topol. 4 (2000), 309-368 (electronic).

[16] P. Lisca. On lens spaces and their symplectic fillings. Math. Res. Lett., 11(1) (2004), 13-22.

[17] P. Lisca and G. Matić, Tight contact structures and Seiberg-Witten invariants, Invent. Math., 129(3) (1997), 509-525.

[18] D. McDuff, The structure of rational and ruled symplectic 4-manifolds, J. Amer. Math. Soc. 3(3) (1990), 679-712.

[19] H. Ohta and K. Ono, Symplectic 4-manifolds containing singular rational curves with (2,3)-cusp, Sémin. Congr., 10 (2005), 233-241.

[20] B. Ozbagci and A. Stipsicz, 'Surgery on contact 3-manifolds and Stein surfaces', Bolyai Society Math. Studies, 13, Springer-Verlag, Berlin, 2004.

[21] S. Schönenberger, Planar open books and symplectic fillings, PhD thesis, University of Pennsylvania, 2005.

[22] A. Stipsicz, Gauge theory and stein fillings of certain 3-manifolds, in Proceedings of the Gökova Geometry-Topology Conference, pp. 115-131, 2001.

[23] W.P. Thurston and H.E. Winkelnkemper, On the existence of contact forms, Proc. Amer. Math. Soc. 52 (1975), 345-347. 
[24] I. Torisu, Convex contact structures and fibered links in 3-manifolds, Int. Math. Res. Not. 9 (2000), 441-454.

[25] H. Wu, Tight contact structures on small Seifert spaces. PhD Thesis, MIT, (2004), See http://home.gwu.edu/ haowu/CV.html.

Department of Mathematics

University Of Pennsylvania

Philadelphia, PA

E-mail address: stephans@math.upenn.edu

Received 12/28/2006, accepted 05/25/2007

The work in this paper is part of the author's $\mathrm{PhD}$ thesis [21] completed at the University of Pennsylvania in 2005. The author deeply greatful to his advisor John Etnyre for his constant guidance and kind support. Special thanks to Dusa McDuff for a conversation during the FRG-workshop in May 2005 at University of Pennsylvania. 
